\title{
Key Transcription Factors Linking Macular Degeneration and Alzheimer's Disease
}

\author{
Joseph W Eichenbaum* \\ Department of Ophthalmology and Pharmacology, Icahn School of Medicine at Mount Sinai, USA
}

*Corresponding author: Joseph W Eichenbaum, Department of Ophthalmology, Icahn School of Medicine at Mount Sinai, Gustav Levy Place, NY, NY 10029, USA.
Received Date: January 01, 2019

Published Date: February 19, 2019

\begin{abstract}
Alzheimer's disease (AD) and age-related macular degeneration (AMD) have certain pathologic features in common. Chronic oxidative stress and neuroinflammation result in aggregate protein deposits, extracellular drusen in AMD, extracellular and intracellular amyloid in $\mathrm{AD}$, and intracellular tau in $\mathrm{AD}$, and mitochondrial proteosomal pathway damage in both. Along with risk factors: aging, smoking, hypertension, hypercholesterolemia, obesity, arteriosclerosis, unhealthy diet, chronic anticholinergic use, and latent herpetic infection, three transcription factors, NRF-2 (nuclear factor-erythroid 2) and NFKB (nuclear factor kappa B) which regulate cellular detoxification from oxidative stress and innate cellular immunity, respectively, and PGC-1 $\alpha$ (peroxisome receptor gamma coactivator), which is the master of mitochondrial biogenesis and antioxidant control, seem to play a major role in disease progression of AMD and AD . Neuropathology and protein marker changes related to imbalances in NRF-2, NFKB, and PGC$1 \alpha$ illustrate neurodegenerative and vision loses commensurate with NRF-2 and PGC- $1 \alpha$ deficiencies and NFKB excess. Examining these transcription factors in more detail may provide insights into slowing the progression of AD and AMD.
\end{abstract}

\section{Introduction}

The global prevalence of AMD in 2020 is projected to be 196 million people [1] and that of Alzheimer's disease was 50 million in 2017 [2]. Chronic oxidative stress and neuroinflammation from aging, injury, and individual risk factors (smoking, hypertension, arteriolosclerosis, obesity, dietary indiscretion, chronic anticholinergic use, and latent infection) in AMD and AD contribute to toxic protein deposits, loss of homeostatic protein clearance, and progressive neurodegeneration [3-11].

Neuroinflammation also provokes persistent immune response, which participates in further brain and macular damage. However, studies using anti-inflammatory therapy in AD and in AMD have yielded small, mixed or inconclusive results [12-15].

Toxic protein accumulation: in AMD, drusen, (lipoprotein deposits between the basal lamina and the retinal pigment epithelial layer, RPE) and lipofuscin (from inefficient protein clearance) $[3,4,13]$ and in AD, extracellular and intracellular amyloid and complement and intracellular tau, (because of breakdown of the blood-brain barrier from reactive oxygen species, inflammation, or local or systemic infection), may be slow burners in chronic inflammation and its sequelae $[5,6,16]$ However, cognitive issues, (memory and learning) in AD may precede amyloid plaque and tau fibrillar aggregates by months or longer [5]. For that matter, in AMD despite impaired lysosomal degradation, lipofuscin accumulation, defects in the ubiquitin protein clearance, and mitochondria dysfunction [3], many ophthalmologists can attest to the fact that visual loss may take years to manifest itself clinically. So, the relationship of the protein deposits to the chronology of clinical AD and AMD is not quite linear.

Similarly, in $\mathrm{AD}$, the ubiquitin, functioning as beta amyloid "gatekeeper", controlling beta amyloid traffic from intracellular compartments to the cell surface is affected by higher levels of beta amyloid cleaving enzyme (BACE) and affects amyloid levels and proteosomal stability and function [3]. So, both in AMD and AD declining mitochondrial function, proteosomal protein degradation and clearance can result in more profound aging and degenerative changes associated with greater homeostatic imbalance. (Figure 1) However, are these associated intracellular ageing breakdown phenomenon or root causes of AD and AMD?

(Figure 1) RPE, retinal pigment epithelium and neurons in AD, Alzheimer's disease and AMD, age related macular degeneration. 


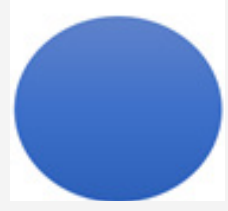

Mitochondria: e-chain, TCA cycle, ATP, Noxa A1 [NADPH], Ucp3, Calcium, Hi/Lo Energy, Respiration, Metabolism, Mito DNA < with age, Ox /Stress/Anti-oxidants, Mito proteins +lipids, Damage, Repair, Mitophagy, Autolysosome, Bcl2 (for Caspace and Apoptosis).

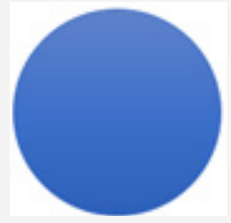

Miscoded, Misfolded, Aggregated proteins, Lipoproteins, lipofuscin, drusen (AMD), complement, Tau, amyloid, (AD) adducts, antibodies, autoantibodies, Autophagy Lysosome Pathway, Autophagosome, Lysosome,



Modifiers: (1) Sirtuins 1,2: up-regulator, down-regulator of acetylation/deacetylation and other epigenetic histone stabilizers; (2) Transcription

Factors: NRF-2, NFKB, and PGC-1a: major protective framework oxidative stress and inflammation.

Figure 1: RPE and Neurons in AMD and AD.

Top Blue Sphere: Mitochonidria: Early Activities in Oxidative Stress and Inflammation

Middle Blue Sphere: Intermediate Activities in Oxidative

\section{Stress and inflammation}

Bottom Blue Sphere: Key Modifiers of Chronic Oxidative

\section{Stress and Inflammation}

Lipofuscin is a highly cross-linked extracellular aggregate of oxidized proteins and lipids from mitochondrial damaged sites, which is not incorporated or degraded by proteasome or lysosomal enzymes but rather itself incorporates transition metals like iron. The iron through the Fenton reaction generates its own free radicals, which induce further retinal mitochondrial dysfunction, malondialdehyde, 4-hydroxynonenal (HNE) and advanced glycation products which enhance RPE aging and photoreceptor degeneration. [3]. In addition, lipofuscin proteins, such as malondialdehyde and HNE, which are markers of oxidative mitochondrial dysfunction, have been shown to provoke auto-immune inflammatory reaction in the retina $[3,17]$. AD patients also have striking mitochondrial dysfunction, (increase in mitochondrial DNA levels) and vacuoles with lipofuscin in the neuronal cytoplasm $[3,18]$. Along similar lines in AD brains, malondialdehyde on electron microscopy formed "cap-like linear deposits" associated with intracellular neuronal lipofuscin $[3,19]$.

In $\mathrm{AD}$, oxidative stress markers, (lipid peroxidation, nitration, reactive carbonyls, and nucleic acid oxidation products) are elevated decades prior to amyloid deposition [3,20,21]. Increased isoprostanes (early products of unsaturated fatty acid oxidation) $[3,22,23]$ and, in other studies, iron has been reported in the cortex and cerebellum of preclinical AD/MCI (Alzheimer's disease mild cognitive impairment) patients $[3,24,25]$ in association with mitochondrial dysfunction [25]. As in AMD, patients with $\mathrm{AD}$ also seem to develop an early imbalance in iron homeostasis and mitochondrial dysfunction, [3,21-25] preceding advanced neurodegenerative change.

Thus, looking at $\mathrm{AD}$ and $\mathrm{AMD}$ from the standpoint of cumulative chronologic protein deposits of a toxic nature in the setting of mitochondrial dysfunction and impaired proteosomal clearance, there are striking similarities but as to the onset of cognitive and vision problems, it might be reasonable to view the signatures of three key protective transcription factors, NRF-2, (oxidative stress and inflammation), PGC- $1 \alpha$ (drives mitochondrial biogenesis and antioxidant defense), and NFKB, (which controls innate immune response) for more detailed understanding of the chronology, genetics and epigenetic aspects of these diseases and their relationship to functional loss. (Figure 1) There is a recent literature with this approach, which will be the focus of this paper.

\section{Materials and Methods}

To gather data on molecular mechanisms of macular degeneration and Alzheimer's disease with respect to: definition, pathology, molecular structures of deposits, proteomics, transcription factor relationships, and homeostatic mechanisms individual and combination search meshes were run in pub med and or Google Scholar with the pertinent terms. In cases where there were differences of interpretation, additional papers were sought to try to reconcile these differences.

\section{Results}

\section{PGC-1 $\alpha$ and NRF-2 loss and AMD}

In RPE cells, the NRF-2/ARE (nuclear factor erythroid 2/ antioxidant response element) regulates oxidative stress by, amongst other things, enabling nuclear antioxidant gene activity and antioxidant proteins $[3,26]$. Mice models with NRF-2 deficiency are more susceptible to oxidative damage, protein aggregation, and poor regulation of autophagy genes $[27,28]$.

Genetic ablation of NRF-2 and PGC- $1 \alpha$ in mice has produced RPE (Retinal pigment epithelial) degeneration [4,29,30]. Knock out of both NRF-2 and PGC-1 $\alpha$ also revealed profound parametric increases in: (1) ubiquitin, a key player in damaged protein removal, (2) 4-HNE, an important marker of oxidative stress, (3) damaged mitochondria, (higher numbers seen on transmission electron microscopy, TEM), (4) p62, which NRF-2 targets to reduce intracellular oxidative stress and inflammation, (5) autolysosome size (on TEM), which help remove damaged proteins, and (6) number of melanosomes, (RPE degradation products from reduced proteasome activity (as seen on TEM). [4] The insufficient rate of autophagic clearance of damaged proteins along with the higher ratio of dysfunctional mitochondria are significant contributors to AMD. $[4,30,31]$ AMD severity has been correlated to mitochondrial DNA damage [30-32]. 
The PGC-1 family regulates mitochondrial: biogenesis, respiration, and antioxidant defense [33-35]. Down regulation or loss of PGC- $1 \alpha$ increases mitochondrial damage. Up regulation increases anti-oxidant genes such as SOD 2 [33-35]. The net goal of PGC- $1 \alpha$ is for mitochondrial homeostasis $[33,35]$.

Thus, the Nrf-2/PGC-1 $\alpha$ double knock out mice had additive pathological changes of each transcription factor deficiency mimicking the presentation of AMD. There was: intra- and extracellular deposit accumulation, increased lipofuscin, Bruch's membrane defects, immune cell invasion, damaged mitochondria, impaired autophagy, altered pigmentation, focal photoreceptor degeneration, and ERG b wave amplitude reduction (showing effect on vision) [4].

The role of the PGC- $1 \alpha$ and Nrf- 2 knockouts in delineating many of the crucial parameters of AMD does not belie the potential contribution of genome wide [36-39] and population studies [40] (and other mice knockout studies [41] illustrating the role of complement factor $\mathrm{H}$ and I and HTRA in attenuating or adding to the evolution of AMD in different settings (Figure 2). With all these genes and yet others, their relationship to age, ethnicity, epigenetics, individual penetrance and protein aggregation patterns is quite enigmatic and still evolving in our understanding of AMD. Diet and exercise and other epigenetic modifiers and CFH genes may have considerable individual sparing effects in the penetrance of AMD.

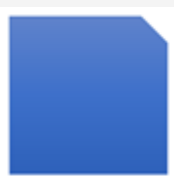

$\mathrm{AD}$ and $\mathrm{AMD}$ genes in common $\mathrm{CFH}$ (regulates complement) and APOE (regulates lipoprotein binding to target receptors. Transcription Factors: NRF-2, PGC-1a, and NFKB: Core antioxidant, Antinflammatory, and Innate immune responses.

\section{AD, AMD Genome Associations}



AD genes: Strongest Association: APOE 4, Clusterin, (binds amyloid and reduces amyloid fibril formation) Clathrin assembly protein. AMD genes most strongly associated: CFH $(402 \mathrm{H})$ protective gene, APOE 2, (risk factor: increases cholesterol in retinal pigment epithelial cells) HTRA (key role in reducing heat shock oxidative stress proteins).

\section{AD, AMD proteins in common}

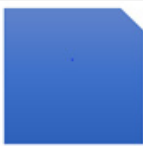

$A D$ and $A M D$ protein deposits in common: Lipofuscin (mitochondrial-proteosomal defects result in deposits). Amyloid (misfolded protein thought responsible for AD, but also protective against Oxidative stress and infections).

Complement (innate immunity; defense against microorganisms, inflammation, And cell destruction)

Figure 2: Genetics and Proteomics of AD and AMD.

\section{NRF-2 loss and AD}

Although the brain expresses low basal levels of NRF-2, even subtle changes in NRF-2 related genes may have large impact on pathophysiological pathways of AD. For example, NRF-2-encoding NFE212 haplotypes influence AD progression [5,42].

Combining NRF-2 deficiency with both amyloidopathy and tauopathy in mice, Rojo et al made a model that would mirror NRF-2 knock out with a combination of both hallmarks of human AD. However, they indicated that it is not clear what the mutual relationship of amyloidopathy and tauopathy is or what the contribution of each is to the idiopathic or sporadic forms of $\mathrm{AD}$ [5].

Nonetheless, Rojo et al did observe that the hippocampus of the NRF-2 knockout with amyloidopathy and tauopathy exhibited greater levels of oxidative and pro-inflammatory markers than controls early on (within the first 6 months). They pointed out this might be similar to early cognitive impairment, from early oxidative and inflammatory stress (without amyloid and tau deposition) in human $\mathrm{AD}[5,43]$. In the 6-month NRF-2 knockout mice (above), acquisition and retention memory (unable to retain memory of a swim platform) was impaired even without amyloid or tau pathology deposits [5,44].

In view of the high impact of well-established familial AD genes, (Apoe 4, APP. PS1 and PS2), in the population, NRF-2 deficiency, which may have a small prevalence in younger $\mathrm{AD}$ patients, where presumably there are higher levels NRF-2 as compared to the aged, there may be other epigenetic, genetic or homeostatic factors operative in the protective mode. However, in the aged, where NRF-2 declines, the consequences of MCI and early AD are more penetrant $[5,45,46]$.

NRF-2 deficient mouse brain with amyloidopathy and tauopathy replicated gene pathway alterations found in human elderly and AD brains. For example, Gstm1 (detoxification and oxidative stress), Kras (protein signaling), and Wdfy1 (vesicular traffic and autophagy), all regulated by NRF-2 and involved in cell signaling and inflammation, were all down regulated in the NRF-2 knockout mice. They also found Zbtb21/ANF295 upregulated, which has been implicated in Down's syndrome and cognitive deficit. The genes studied were classified in functional clusters of biological processes by GOTERM_BP_FAT, which examines the significance of enrichment of functional annotations. The genes were then compared to three cohorts of old human brain and six cohorts of AD brain. All of the NRF-2 knock out mice gene pathways were found altered in human ageing or AD brains, but some of the elderly or AD brain pathways were not found in the knock out mice. This suggests an NRF-2 knock out-group transcriptomic gene participation in the pathological process of $\mathrm{AD}[5]$.

NRF-2 knockout brains had lower levels of reduced glutathione and higher levels of carbonylated proteins (adducts of 2,4 dinitrophenylhydrazine, DPNH) and lipid peroxides (malondialdehyde, MDA) than controls, which point to the consequences of less control of chronic oxidative stress in the knockouts [5]. Proinflammatory markers, IL6, COX2, and iNOS were increased in NRF-2 knockouts compared with controls, consistent 
with the anti-inflammatory protective effect of NRF-2. Thus, both chronic oxidative and inflammatory stress, as well as aging, appear to be more prominent in absence of NRF-2 [5].

Kaplan-Meier survival rates for NRF-2 knockouts with amyloidopathy and tauopathy showed $50 \%$ of the knockouts dying before 12 months, similar in males and females, whereas, controls reached to twenty months [6].

These same knockouts showed increased brainstem glial activation (as evidenced by increased antibody, GFAP and IBA1 staining compared with controls). Thus, NRF-2 deficiency modified immune response [6].

Nine months old NRF-2 knockouts with amyloidopathy and tauopathy mice (of the above noted research) were treated with oral gavage with vehicle or DMF, (dimethyl fumarate, an antioxidative and immunosuppressive) $100 \mathrm{mg} / \mathrm{kg}$ every other day for six weeks. Five days after the first administration the mice were subjected to double blind motor and novel object recognition tests for memory. This was repeated once every two weeks. mRNA levels of NRF-2 target genes were analyzed by qRT-PCR.

There was a statistically significant: (1) increase in the levels of NRF-2 knock outs with DMF gavage of Ngo1, Osgin1, and Gstm1 (three oxidative stress detoxification genes), (2) reduction of astrocytosis and microgliosis (using GFAP and IBA1 antibodies in hippocampus and brainstem neurons), (3) reduction of protein levels of inflammation (less pro-inflammatory COX2, NOS2), (4) increased motor scores, and (5) increased novel object recognition scores by 2-3 fold, all in DMF gavage treated NRF-2 knockouts amyloidopathy tauopathy mice versus vehicle treated. This would suggest not only the potential role of DMF for NRF-2 activation when it is deficient in $\mathrm{AD}$, but also that brain oxidative and inflammation stress, which correlates to motor, memory and immune consequences might be attenuated from full expression over time in AD with the use of pro-NRF-2 stimuli such as DMF [6].

\section{PGC-1 $\alpha$, Mitochondrial Biogenesis and AD}

PGC- $1 \alpha$, which is found in tissues with high-energy demand $[47,48]$, such as muscle and brain, exhibits decreased expression in the brains of $\mathrm{AD}$ patients $[49,50]$. PGC- $1 \alpha$ forms a heteromeric complex with NRF-2 and other transcription factors [51] in response to environmental stress, temperature, and nutritional status, (including insulin resistance) and could displace repressor proteins such as histone de-acetylase [52] and induce gene activation for reactive oxygen species detoxification, such as superoxide dismutase 1 and 2 , catalase and glutathione peroxidase [53] PGC- $1 \alpha$ also modulates the expression of uncouple protein 2 and uncouple 3 (See Figure 1, Ucp3, under mitochondria) which are primers of reactive oxygen species formation [54,55]. Similarly, PGC- $1 \alpha$ controls sirtuin 1 and sirtuin 3 which reduce reactive oxygen species formation [56-58].

Neurodegeneration and synaptic degradation in AD are strongly tied to impaired mitochondrial biogenesis [59]. Mitochondrial impairment is central to the progression of $\mathrm{AD}$ and neuronal apoptosis, $[60,61]$ both from the standpoint of loss of energy metabolism and excess free radical formation and damage [62-66]. Several human and animal studies have correlated mitochondrial dysfunction and memory difficulties $[67,68]$ In the aged brain, PGC$1 \alpha$ regulates the expression of sirtuin 3 , which is an important factor in the process of aging. Patients with neurodegenerative diseases harbor low levels of PGC-1 $\alpha$, which leads to greater oxidative stress and mitochondrial dysfunction [69,70]. PGC-1 $\alpha$ knockouts mice showed reduction of mitochondrial gene expression and neuronal dysfunction and others have shown that the presence of PGC- $1 \alpha$ could improve mitochondrial dysfunction and cognitive function [71,72].

Thus, because of its manifold anti-oxidant, homeostatic metabolic, mitochondrial biogenesis roles as well as its co-opted protective gene activation roles, PGC- $1 \alpha$ may play a significant role in the understanding of $\mathrm{AD}$ (Figure 2).

\section{(-3 Fatty Acids , NFKB and AMD}

There are several studies [73-75] illustrating the protective role of long chain $\omega-3$ fatty acids against oxidative stress and inflammation in the development of AMD. $\omega$-3 fatty acids are comprised of linoleic acid, docosahexaenoic acid (DHA), and eicosapentaneoic acid (EPA). Linoleic acid (a short chain $\omega$-3 fatty acid), found in e.g. flaxseed oil, is a dietary precursor to DHA and EPA and can be converted by humans to some extent to DHA, a long chain $\omega-3$ fatty acid [73]. Otherwise fish intake provides DHA.

Higher levels of DHA are present in the retinal photoreceptor outer segments than any other tissue [76]. DHA is a central regulator of retinal membrane, visual cycle [77] transport and a precursor to the synthesis of other active molecules [78]. EPA regulates lipoprotein metabolism and suppresses inflammatory compounds that can damage Bruch's membrane and lead to choroidal neovascularization [79].

Resolvens and protectans are biosynthesized from $\omega-3$ fatty acids EPA and DHA via cyclogenase- 2 and are thought to be potent long acting anti-oxidative and anti-inflammatory molecules [80]. Neuroprotectin D, from the protectin D family has anti-apoptotic effects on oxidatively stressed retinal pigment epithelial cells [81]. NFKB protein, one of the cell's master responders to oxidative stress and immune defense [82] is retained in the cytoplasm in an inactive state, bound to inhibitory proteins $\operatorname{IKB} \alpha, \operatorname{IKB} \beta, \mathrm{IKB} \Upsilon \mathrm{IKB} \varepsilon$ and $\mathrm{Bcl} 3$, also known as IKB. After oxidative stress, IKB kinase (IKK) is activated, which phosphorylates IKB proteins rendering them responsive to the ubiquitin system for protein degradation [83]. More important, however, the NFKB is moved to the nucleus where it forms activation complexes to promote various target genes which regulate acute and chronic inflammation, such as TNF $\alpha$, IL1, IL-2, IL-6, IL-8, IFNY, inducible NO synthase, adhesion molecules, cyclogenase- 2 and CRP (c reactive protein, produced in the liver and elevated in the blood in response to acute inflammation) [82,83].

The $\omega$-3 fatty acids can block key receptors, such as Toll 4, TNF, and prevent IKB kinase phosphorylation and attenuate exuberant NFKB transcription factor anti-inflammatory activity in RPE cells, (oxidative stress, protein misfolding and aggregation, mitochondrial/proteosomal overload, drusen, lipofuscin and 
RPE/photoreceptor apoptosis) which lead to an aging macula and macular degeneration $[73,84]$. Thus, $\omega-3$ fatty acids can act as a brake on the excesses of NFKB response to oxidative stress, inflammation, aging and dry AMD.

\section{NVKB and AD}

The recent failure of another anti-amyloid treatment in large phase III trials for AD has suggested that alternate hypothesis for neuro-protection are necessary [85]. Beta amyloid production as an antimicrobial peptide, following exposure to neurotoxic fungi, in cell lines, nematode and rodent models [86] may be a byproduct of latter pathway immune dysregulation rather than the disease process itself. Several genes involved in innate immunity and associated with an increase in $\mathrm{AD}$ speak to this counter point through NFKB [87] For example, late AD onset gene TREM2, which is required for microglial amyloid clearance, is suppressed in the hippocampus by NFKB [88]. CD33, which inhibits microglial beta amyloid uptake and clearance, activates NFKB in myeloid cells [89]. CR1, whose interaction with amyloid is uncertain, is activated by microglia and is associated with increased NFKB [90]. So NFKB levels or gene/protein pathways, (in addition to or in conjunction with those of amyloid may represent a more complete method for understanding $\mathrm{AD}$ ) and as illustrated above, may vary according to microglial, beta amyloid, and protein clearance needs, but may also be under other alternate varied gene control for anti-inflammatory/ oxidative stress activities (Figures $1 \& 2$ ).

Thus, NFKB is the intra-cellular trigger sensitive point for manifold activators from aging, to oxidative stress, to bacterial lipopolysaccharides, to ionizing radiation, to growth factors, to glutamate, etc. which converge on IKK kinase and result in innate immune response. The NFKB entourage sets the stage for a variety of new pathways of exploration of AD [91].

In humans, overexpression of amyloid precursor protein APP is associated with late onset $\mathrm{AD}$ and increases the likelihood of early AD in Down's syndrome. Beta secretase, BACE 1, cleaves APP into amyloid monomers that go on to form oligomers and plaque in neurons and vessels. BACE 1 and NFKB are increased in brains with $\mathrm{AD}$, and NFKB directly increases BACE and APP gene activity $[92,93]$.

Apoe 4 gene, which codes for cholesterol transporting protein, and is the single largest gene risk factor for $\mathrm{AD}$, acts as a transcription factor for regulating NFKB expression [94]. Curiously, in early AD, the somatosensory cortex may show upregulation of NFKB, but there is reduction of NFKB in areas of more mature plaque $[95,96]$. So, there is a variable dynamic in NFKB expression not only relative to the presence of pathology, but also related to the time of onset and the evolution of the AD changes.

In rats, NFKB expression increases in normal aging, leading to production of neurodegenerative pro-inflammatory COX2 and iNOS enzymes [97]. However, using anti-inflammatory Lactobacillus pentosus, plantarum C29, suppresses NFKB and restores brain derived neurotrophic factor levels and memory [98]. In rats exposed to neurotoxin, NFKB induced pro-apoptotic increases in TNF and iNOS in the hippocampus [99].
In mice, inactivating anti-aging Sirtuin genes results in chronic NFKB over expression, which significantly reduces the lifespan [100] Age related NFKB activation leads (through the chronic inflammation in microglial cells) to among other responses, epigenetic suppression of $\mathrm{GnRH}$ genes (gonadotropin releasing genes) in the hypothalamus [101] So NFkB with its manifold activities from amyloid production and plaque formation to glial activation, and aging seems to be vital to neurological rebalancing against the challenges of chronic oxidative stress, inflammation and protein aggregation in the development of AD.

\section{Cross Talk between NRF2 and NFKB}

Frequent changes in the levels of oxidative stress and inflammatory response necessitate an ongoing dynamic in two key cellular transcription factors NRF-2 and NFKB. Pharmacological and genetic inquiries suggest that there is a purposeful cross talk between these transcription factors leading to positive and negative effects on target gene expression. The absence of NRF2 can raise NFKB activity leading to increased cytokine production, astrogliosis, demyelination, and neuronal cell death [102-104]. Aside from its regulatory role in NRF-2-ARE pathway (nuclear active response element), Keap1 can negatively regulate IKK $\beta$, (an essential NFKB precursor) by blocking HSP90 (heat shock protein 90 ) binding to IKK $\beta$ and retain lower NFKB levels of activity [105].

Both NRF-2 and NFKB are also connected through $\beta$-TrCP, a component of the E3 ligase complex (responsible for clearance of toxic proteins to proteasomes). $\beta$-TrCP controls nuclear levels of NRF-2 [106,107] The same kinase that marks NRF-2 for $\beta$-TrCP binding, GSK3 $\beta$, is also involved in NFKB DNA binding [108,109]. In addition, since $\beta$-TrCP regulates NFKB response to cytokines as well as inhibition of NRF2 transcription, it can have an up-regulation effect on NFKB and a down regulation effect on NRF-2 [110]

P62, another NRF-2 target gene, modulates intracellular anti-inflammatory and anti-oxidant activities. P62, acting as protein scaffolding, can increase NRF-2 activity by mediating autophagosomal breakdown of Keap1, the key NRF-2 regulator [111] However p62 also has the ability to activate TNF $\alpha$, which is a key enabler of NFKB [112]. There are also other interactions of NRF- 2 and NFKB [113]. Thus, the cross talk can result in the activity of both NFKB and NRF-2 or limit the activity of one in favor of the other.

\section{Discussion}

The development and use of NRF-2 and PGC- $1 \alpha$ mouse knockouts to study AMD [4] and NRF-2 with amyloid and tau pathology knockouts to examine $\mathrm{AD}[5,6]$, provide powerful tools in our understanding of NRF-2 and PGC- $1 \alpha$ in neurodegenerative disease. In addition to investigating protein aggregation, autophagy, proteosomal clearance, mitochondrial dysfunction, immune reaction and genomic markers after oxidative stress and chronic inflammation, the knockouts were also monitored for vision (ERG in the AMD study [4]) and motor and memory [5,6] in the AD study. Similarities of the mouse knockout studies to each other as well as to their respective comparisons to human AMD and AD are intriguing. 
The highest increase in protein aggregation and oxidative stress markers in the AMD NRF-2/ PGC-1 $\alpha$ knock out study [4] was with the combination of NRF-2 and PGC- $1 \alpha$ gene knockouts. In the AD knock out studies [5,6] NRF-2 itself (with tauopathy and amyloidopathy) yielded significant gliosis, motor, cognitive and neuroinflammatory changes. Also, in line with other animal models, memory and learning were severely impaired as early as 6 months, preceding the appearance of amyloid and tau deposits. NRF2 deficiency in mice was quite similar to transcriptomic features found in human AD brains [6,114]

The protective role of DMF in the NRF-2 deficient mice in reducing oxidative stress and inflammatory gene activity, astrocytosis and gliosis levels, and enhancing motor and memory scores was remarkable [6]. However, in other studies with DMF, the DMF mechanism of action has been attributable to: (1) alternate pathways independent of NRF-2 [115], (i.e.: interferon $\Upsilon$ reduction, IL-17 producing CD4(+) cells and induction of anti-inflammatory M2 monocytes), (2) decreased synthesis of pro-inflammatory iNOS, TNF $\alpha$, IL-1 $\beta$ and IL- 6 and activation of microglia and astrocytes [116] and (3) dimethyl fumarate causes short-lived oxidative stress, which leads to increased levels and nuclear localization of NRF-2 and a subsequent increase in glutathione synthesis and its recycling in neuronal cells [117] Future investigation examining the effects of DMF as an anti-inflammatory agent in the NRF-2 and deficient NRF2 setting is indicated.

SIRT1 is a quiet information regulator and the mammalian homolog of the nicotinamide adenine dinucleotide, NAD deacetylase. In addition to histones for epigenetic alterations in gene expression, SIRT1 targets include transcription factors such as NFKB and PGC- $1 \alpha$. In vitro and in vivo studies had revealed the beneficial role of SIRT1 in regulating neural progenitors, axon elongation, dendritic branching, synaptic plasticity and endocrine function. SIRT1 has been implicated in neuroprotection in AD and traumatic brain injury [118]

Although the role of NRF-2, PGC- $1 \alpha$, and NFKB has been discussed here in trying to understand a more broad genomic reach of transcription factors in AMD and AD, how gene activity evidenced through genome wide association or population studies, such as complement factor $\mathrm{H}$ or APOE, and even epigenetic modifiers and Sirtuins tie in with transcription factors is as yet to be elicited It is possible that an individual's transcription factors act as a metabolic/energy framework to protect the individual through the oxidative and inflammatory challenges, (smoking, dietary, exercise, sleep, infection, and adverse inherited genes) and provide some measure of a dynamic re-balancing to sustain the organism.

\section{Conclusion}

Because of their over-arching presence in the landscape of the neuro-protective/neurotoxic terrain in $\mathrm{AMD}$ and $\mathrm{AD}$, the transcription factors NRF-2, PGC1 $\alpha$, and NFKB represent an alternate approach to understanding these diseases. Whether the new ideas on management derive from re-tooling anti-oxidative stress players, (e.g. up-regulating NRF-2 and/or PGC- $1 \alpha$ or their more relevant target genes) in AMD or resetting the proinflammatory and immune consequences of NFKB and e.g. TNF in early $\mathrm{AD}$, more in depth research of the transcription factors activity in each disease will provide greater understanding of the complex details necessary for alternate therapeutic strategies.

\section{Acknowledgement}

I would like to thank Dr. Martin J. Walsh, Professor Pharmacological Sciences, Pediatrics, Genetics and Genomic Sciences and Dr. Ming Ming Zhou, Professor and Chair Pharmacological Sciences and Co-director Experimental Therapeutics Institute: Mount Sinai School of Medicine for their many hours of enlightening conversations and strategic input on genomics and epigenetic mechanisms over the last decade.

\section{Conflicts of Interest}

No conflicts of interest.

\section{References}

1. Wong WL, Su X, Li X, Cheung CM, Klein R et al. (2014) Global Prevalence of age-related macular degeneration and disease burden projection for 2020 and 2040: a systematic review and meta-analysis. The Lancet Global Health 2(2): e106-e116.

2. Dementia Statistics Alzheimer's disease International. World Report 2015.

3. Kaarniranta K, Salminen A, Haapasalo A, Soininen H, Hiltunen M (2011) Age related macular degeneration: Alzheimer's disease of the eye. Journal of Alzheimer's Disease 24(4): 615-631

4. Felszeghy S, Viiri J, Paterno JJ, Juha MT Hyttinen, Ali Koskela et al. (2019) Loss of NRF-2 and PGC- $1 \alpha$ genes leads to retinal pigment epithelium damage resembling dry age-related macular degeneration. Redox Biology 20: 1-12.

5. Rojo AI, Pajares M, Rada P Nuñez A, Nevado Holgado AJ et al. (2017) NRF2 deficiency replicates transcriptomic changes in Alzheimer's patients and worsens APP and TAU pathology. Redox Biology 13: 444451.

6. Rojo AI, Pajares M, Garcia Yague AJ Buendia I, Van Leuven F et al. (2018) Deficiency in the transcription factor NRF2 worsens inflammatory parameters in a mouse model with combined tauopathy and amyloidopathy. Redox Biology 18: 173-180.

7. Aldebert G, Fallie JL, Hillaire Buys D Mura T, Carrière Iet al. (2018) Association of anticholinergic drug use with risk for late age -related macular degeneration. JAMA Ophthalmology 136(7): 770-778.

8. Fierz W (2017) Age related macular degeneration: a connection between human herpes virus $6 \mathrm{~A}$ induced $\mathrm{CD} 46$ downregulation and complement activation? Front Immunol 8: 1314

9. Readhead B, Haure Mirande JV, Funk CC, Richards MA et al. (2018) Multiscale analysis of Independent Alzheimer's cohorts finds disruption of molecular, genetic and clinical networks by human herpes virus. Neuron 99(1): 64-82.

10. Kalayoglu MV, Galvan C, Mahdi OS, Byrne GI, Mansour S et al. (2003) Serological association between Chlamydia pneumonia infection and age-related macular degeneration. Archives of Ophthalmology 121(4): 478-482.

11. Miller DM, Espinosa Heidmann J, Legra, Dubovy SR, Sũner IJ et al. (2004) The association of prior cytomegalovirus infection with age related macular degeneration. American J of Ophthalmology 138(3): 323-328.

12. Li S, Hu A, Wang W, Ding X1, Lu L (2017) Combinatorial treatment with topical NSAID and anti-VEGF for age related macular degeneration, a meta-analysis. Plos one 12(10): e0184998.

13. Ambati J, Fowler B (2012) Mechanisms of age- related macular degeneration. Neuron 75(1): 26-39.

14. De Craen AJ, Gussekloo J, Vrijsen B, Westendorp RG (2005) Meta-analysis of non-steroidal anti-inflammatory drug use and risk of dementia. Am J Epidemiology 161(2): 114-120. 
15. Int Veld BA, Ruitenberg A, Hofman A Launer LJ, Van Duijn CM et al. (2001) Non-steroidal anti-inflammatory drugs and the risk of Alzheimer's disease. NEJM 345(21): 1515-1521.

16. Morgan PB (2018) Complement in the pathogenesis of Alzheimer's disease. Semin Immunopath 40: 113-124.

17. Gu X, Meer SG, Miyagi M Rayborn ME, Hollyfield JG et al. (2003) Carboxyethylpyrrole protein adducts and autoantibodies, biomarkers for age related macular degeneration. J of Biological Chemistry 278 42027-42035.

18. Hirai K, Aliev G, Nunomura A Fujioka H, Russell RL et al. (2001) Mitochondrial abnormalities in Alzheimer's disease. J Neurosci 21(9): 3017-3023.

19. Dei R, Takeda A, Niwa H, Li M, Nakagomi Y et al. (2002) Lipid peroxidation and advanced glycation end products in the brain in normal aging and in Alzheimer's disease. Acta Neuropathol 104(2):113-122.

20. Aluise CD, Robinson RA, Becket TL (2010) Preclinical Alzheimer disease: brain oxidative stress. Abeta peptide and proteomics. Neurobiol Dis 39(2): 221-228.

21. Zhu X, Raina AK, Perry G, Smith MA (2004) Alzheimer's disease: the twohit hypothesis. Lancet Neurol 3(4): 219-226.

22. Pratico D, Lee WM, Trojanowski JQ Rokach J, Fitzgerald GA (1998) Increased F2-isoporstanes in Alzheimer's disease: evidence for enhanced lipid peroxidation in vivo. FASEB J 12(15): 1777-1783.

23. Pratiaco D, Clark CM, Liun F, Rokach J, Lee VY, et al. (2002) Increase of brain oxidative stress in mild cognitive impairment; a possible predictor of Alzheimer's disease. Arch Neurol 59(6): 972-976

24. Smith MA, Zhu X, Tabaton M, Liu G, McKeel DW, et al. (2010) Increaed iron and free radical generation in preclinical Alzheimer's disease and mild cognitive impairment. J Alzheimers Dis 19(1): 363-372.

25. Moreira PI, Carvalho C, Zhu X, Smith MA, Perry G (2010) Mitochondrial dysfunction is a trigger of Alzheimer's disease pathophysiology. Biochim Biophys Acta 1802(1): 2-10.

26. Zhao Z, Chen Y, Wang J, Sternberg P, Freeman ML et al. (2011) Agerelated retinopathy in NRF-2 deficient mice. Plos One 6(4): e19456.

27. Pajares M, Jimenez Morene J, Garcia Yague M, Escoll M, De Ceballos ML et al. (2016) Transcription Factor Nff212/NRF2 is a regulator of macroautophagy genes. Autophagy 12(10): 1092-1916.

28. Wei Y, Gong T, Yoshida C, Charles, G Eberhart, et al. (2011) NRF2 has a protective role against neuronal and capillary degeneration in retina ishchemia reperfusion injury. Free Radical Biol Med 51(1): 216-224.

29. Egger A, Samarddzija M, Sothilingam V, Tanimoto N, Lange C, et al. (2012) PGC-1 $\alpha$ determines light damage susceptibility of the murine retina. Plos One 7(2): e31272.

30. Feher J, Kovacs M, Artico C, Cavallotti A, Papale C, et al. (2006) Mitochondrial alterations of retinal pigment epithelium in age related macular degeneration. Neurobiol Aging 27(7): 983-993.

31. Ferrington DA, Kapphahn RJ, Leary MM, Atilano SR, Terluk MR, et al (2016) Increased retinal mDNA damage in the CFH variant associated with age related macular degeneration. Exp Eye Res 145: 269-277.

32. Terluk MR, Kapphahn RJ, Soukup LM, Gong H, Gallardo C, et al. (2015) Investigating mitochondria as a target for treating age related macular degeneration. J Neurosci 35(18): 7305-7311.

33. Baldelli S, Aquillano K, Cirolo MR (2014) PGC-1 $\alpha$ buffers ROS-mediated removal of mitochondria during myogenesis. Cell Death Dis. 5: e1515.

34. Aquilano K, Baldelli S, Pagliei B, Cannata S, Rotillo G (2013) p53 orchestrates the PGC-1a mediated antioxidant response upon mild redox and metabolic imbalance. Antioxid Redox Signal 18(4): 386-399.

35. Marmolino D, Manto M, Acquaviva F Vergara P, Ravella A, et al. (2010) PGC- $\alpha$ down regulation affects the antioxidant response in Friedrich's ataxia. Plos One 5(4): e10025.

36. Edwards O, Ritter R, Abel KJ, Manning A, Panhuysen C, et al. (2005) Complement Factor $\mathrm{H}$ polymorphism and age realted macular degeneration. Science 308(5720): 421-424.
37. Klein RJ, Zeiss C, Chew EY, Tsai JY, Sackler RS, et al. (2005) Complement factor $\mathrm{H}$ polymorphism in age realted macular degeneration. Science 3080(5720): 385-389.

38. Hains JL, Hauser MA, Schmidt S (2005) Complement factor H variant increases the risk of age-related macular degeneration. Science. 308(5720): 419-421.

39. Gorin MB (2012) Genetic insights into age realted macular degeneration: controversies addressing risk, causality and therapeutics. Mol Aspects Med 33(4): 467-486.

40. Yoshimura N (2016) Age related macular degeneration (in Japanese). Nippon Ganka Gakkai Zasshi 120(3): 163-188.

41. Ding JD, Kelly U, Landowski M (2015) Expression of Human Complement factor $\mathrm{H}$ prevents age related macular degeneration-like retina damage and kidney abnormalities in aged Cfh knockout mice. Am J of Pathol 185(1): 29-42.

42. Von Otter M, Landgren S, Nilsson M, Zetterberg M, Celojevic D, et al. (2010) NRF2 encoding NFE212 haplotypes influence disease progression but not risk in Alzheimer's disease and age-related cataract. Mech. Ageing Dev 131(2):105-110.

43. Pratico D (2008) Evidence of oxidative stress in Alzheimer's disease brain and antioxidant therapy: lights and shadows. Ann NY Acad Sci 147: 70-78.

44. Terwel D, Muyllaert I, Dewachter P, Borghgraef P, Croes S, et al. (2008) Amyloid activates GSK-3beta to aggravate neuronal tauopathy in bigenic mice. Am J Pathol 172(3): 786-798.

45. Ungvari Z, Bailey-Downs L, Gautam T, Sosnowska D, Wang M, et al. (2011) Age associated vascular oxidative stress, NRF-2 dysfunction, and NFKB activation in non-human primate Macaca mulatta. J Gerontol A Biol Sci Med Sci 66(8): 866-875.

46. Tomobe K, Shinozuka Y, Kuroiwa M, Nomura Y (2012) Age related changes of NRF-2 and phosphorylated GSK-3beat in a mouse model of accelerated aging (SAMP8). Arch Gerontol Geriatr 54(2): e1-e7.

47. Lai L, Wang M, Martin OJ, Leone TC, Vega RB, et al. (2014) A role for peroxisome proliferator activated receptor gamma coactivator 1 (PGC1 ) in the regulation of cardiac mitochondrial phospholipid biosynthesis. J Biol Chem 278(4): 30843-30848.

48. Pinho RA, Pinho CA, Tromm CB (2013) Changes in the cardiac oxidative metabolism induced by PGC-1a: response of different physical training protocols in infarction induced rates. Int J Cariol 168: 4560-4562.


expression decreases in the Alzheimer disease brain as a function of dementia. Arch Neurol 66(3): 352-361.

50. Zheng B, Liao Z, Locasio JJ, Lesniak KA, Roderick SS, et al. (2010) PGC$1 \alpha$, a potential therapeutic target for early intervention in Parkinson's disease. Sci Transl Med 2(65): 52-73.

51. Lin J, Tarr PT, Yang R, Rhee J, Puigserver P, et al. (2003) PGC-1 beta in the regulation of hepatic glucose and energy metabolism. J Biol Chem 278(33): 30843-30848.

52. Lin J, Handschin C, Spiegelman BM (2005) Metabolic control through the PGC-1 family of transcription coactivators. Cell Metab 1(6): 361-370.

53. St pierre J, Drori S, Uldry M, Silvaggi JM, Rhee J, et al. (2006) Suppression of reactive oxygen species and neurodegeneration by PGC-1 transcriptional coactivators. Cell 127(2): 397-408.

54. Wu Z, Puigserver P, Andersson U, Zhang C, Adelmant G, et al. (1999) Mechanisms controlling mitochondrial biogenesis and respiration through the thermogenic coactivator PGC-1. Cell 98(1): 115-124.

55. Brand MD (2000) Uncoupling to survive? The role of mitochondrial inefficiency in age. Exp Gerontol 35(6-7): 8118-8120.

56. Rodgers JT, Lerin C, Gerhart Hines, Puigserver P (2008) Metabolic adaptations through the PGC- $1 \alpha$ and SIRT1pathways. FEBS Lett 582(1): 46-53

57. Ozden O, Park SH, Kin HS, Jiang H, Coleman MC, et al. (2011) Acetylation of MN SOD directs enzymatic activity responding to cellular nutrient status or oxidative stress. Aging (Albany NY) 3(2): 102-107. 
58. Sahin E, Colla S, Liesa M, Moslehi J, Müller FL, et al. (2011) Telomere dysfunction induces metabolic and mitochondrial compromise. Nature 470: 359-365.

59. Reddy PH, Tripathi R, Troung Q, Tirumala K, Reddy TP, et al. (2012) Abnormal mitochondrial dynamics and synaptic degeneration as early events in Alzheimer's disease: implication to mitochondria targeted antioxidant therapeutics. Biochim Biophys Acta 1822(5): 639-649.

60. Sweeney G, Song J (2016) The association between PGC-1 $\alpha$ and Alzheimer's disease. Anatomy and Cell Biology 49(1): 1-6.

61. Obulesu M, Lakshimi MJ (2014) Apoptosis in Alzheimer's disease: an understanding of the physiology, pathology and therapeutic avenues. Neurochem Res 39(12): 2301-2312.

62. Maruszak A, Zekanowski C (2011) Mitochondrial dysfunction and Alzheimer's disease. Prg Neuropsychopharmacol Bio Psychiatry 35(2): 320-330.

63. Picone P, Nuzzo D, Caruana L, Scafidi V, Di Carlo M (2014) Mitochondrial dysfunction: different routes to Alzheimer's disease therapy. Oxid Med Cell Longev 11

64. Atamna H, Kumar R (2010) Protective role of methylene blue in Alzheimer's disease via mitochondria and cytochrome c oxidase. J Alzheimers Dis 2: S439-S452.

65. Zumbnko G, Sauer (1989) P SOD-1 activity and platlet membrane fluidity I Alzheirmer's disease. Biol Psychiatry 25(6): 671-678.

66. Moreira PI, Santos MS, Sena C, Nunes E, Seica R, et al. (2005) CoQ10 therapy attenuates amyloid beta-peptide toxicity in brain mitochondria isolated from aged diabetic rats. Exp Neurol 196(1): 112-119.

67. Bishop NA, Lu T, Yankner BA (2010) Neural Mechanisms of aging and cognitive decline. Nature 464(7288): 529-535.

68. Aksenov V, Long J, Liu J, Szechtman H, Khanna P, et al. (2013) A complex dietary supplement augments spatial learning, brain mass and mitochondrial electron transport chain activity in aging mice. Age (Dordr) 35(1): 23-33.

69. Handschin C, Kobayashi YM, Chin S, Seale P, Campbell KP, et al. (2007) PGC1 $\alpha$ regulates the neuromuscular junction program and ameliorates Duchenne muscular dystrophy. Genes Dev 21(7): 770-783.

70. Cui L, Jeong H, Borovecki F, Parkhurst CN, Tanse N, et al. (2006) Transcriptional repression of $\mathrm{PGC}-1 \alpha$ by mutant huntingtin leads to mitochondrial dysfunction and neurodegeneration Cell 127(1): 59-69.

71. Ma D, LI S, Lucas EK, Cowell RM, Lin JD (2010) Neuronal inactivation of peroxisome proliferator activated receptor gamma coactivator $1 \alpha$ (PGC$1 \alpha$ ) protects mice from diet induced obesity and leads to degenerative lesions. J Biol Chem 285(50): 39087-39095

72. Lin J, Wu PH, Tarr Pt,Lindenberg KS, St-Pierre J et al. (2004) Defects in adaptive energy metabolism with CNS-linked hyperactivity in PGC-1 $\alpha$ null mice. Cell 119(1): 121-35.

73. Ariel A, Serhan CN (2007) Resolvens and protectins in the termination program of acute inflammation. Trends Immunol 28(4): 176-183.

74. Hasturk H, Kantarci A, Gouget Sumenian E, Blackwood A, Andry C, et al. (2007) Resolvin E1 regulates inflammation at the cellular and tissue level and restores tissue homeostasis in vivo. J Immunol 179(10): 70217029 .

75. Schwab JM, Chiang N, Arita M, Serhan CN (2007) Resolving E1 and protectin D1 activated inflammation resolution programmes. Nature 447(7146): 869-874

76. StoneWL, Farnsworth CC, Dratz EA (1979) A reinvestigation of the fatty acid content of bovine, rat, and frogretina rod outer segments. Exp Eye Res 28(4): 387-397.

77. Gibson NJ, Brown MF (1993) Lipid headgroup and acy chain compositon modulated the MI-MII equilibrium of rhodopsin in recombinant membranes. Biochemistry 32(9): 2438-2454.

78. Kaarniranta K, Salminen A (2009) NFKB signaling as a putative target for omega -3 metabolites in the prevention of age realted macular degeneration. Experimental Gerontology 44(11): 685-688.
79. Chong EW, Kreis AJ, Wong TY, Simpson JA, Guymer RH Dietary omerg-3 fatty acid and fish intake in the primary prevention of age realted macular degeneration: a systematic review and meta-analysis. Arch Ophthalmol 126(6): 826-833.

80. Serhan CN, Chiang N, Van Dyke TE (2008) Resolving inflammation: dual anti-inflammatory and pro-resolution lipid mediators. Nat Rev Immunol 8(5): 349-361.

81. Mukherjee PK, Marchesilli VL, Barreiro S, Hy J, Bok D, et al. (2007) Neurotrophins enhance retinal pigment epithelial cell survival through neuroprotectin D1 signaling. Proc Natl Acad Sci USA 104(32): 1315213157.

82. Salminen A, Ojala J, Huuskonen J, Kauppinen A, Suuronen T, et al. (2008) Interaction of aging associated signaling cascades: inhibition of NFKB signaling by longevity factors FoxOs and SIRT1. Cell Mol Life Sci 65(6-7): 1049-1058.

83. Barnes PJ, Karin M (1997) Nuclar factor kappa B: a pivotal transcription factor in chronic inflammatory diseases. NEJM 336: 1066-1071.

84. Chung HY, Cesari M, Anton S, Marzetti E, Giovannini S, et al. (2009) Molecular Inflammation: underpinnings of aging and age-related diseases. Ageing Res Rev 8(1): 18-30.

85. Hawkes N (2016) Promise of new Alzheimer's drug is dashed after lack of evidence. BMJ 355: i6362.

86. Vijaya Kumar DK, Choi SH, Washicosky KJ et al. (2016) Amyloid beta peptide protects against microbial infection in mouse and worm models of Alzheimer's disease. Sci Transl Med 8(340): 340-372.

87. Robinson M, Brenda Y, Lee A, Francis T, Hane J (2017) Recent progress in Alzheimer's disease research, pat 2; genetics and epidemiology. Alzheimer Dement 57(2): 317-330.

88. Zhao Y, Bhattacharjee S, Jones BM, Prerna Dua D, Peter N Alexandrov, et al. (2013) Regulation of TREM2 expression by an NFKB sensitive miRNA-34a. Neuroreport 24(6): 318-323.

89. Grciuc A, Serrano Pozo A, Parrado AR, Lesinski AN, Asselin CN, et al. (2013) Alzheimer's disease risk gene CD33 inhibits microglial uptake of amyloid beta. Neuron 78(4): 631-643.

90. Zhu XC, Yu JT, Jiang T, Wang P, Cao L, et al. (2015) CR1 in Alzheimer's disease. Mol Neurobiol 51(2): 753-65.

91. Jones SV, Kounatidis (2017) Nuclear Factor Kappa B and Alzheimer's disease, unifying genetic and environmental risk factors from cell to humans. Frontiers in Immunology 8: 1805.

92. Grilli M, Ribola M, Alberici A, Valerio A, Memo M, et al. (1955) Amyloid precursor protein APP gene expression is controlled by NFKB/ Rel protein. In: HaninI, Yoshida M, Fisher A (eds). Alzheimer's and Parkinson's Diseases Part of the Advances in Behavioral Biology Book Series ABBI US: Springer, USA, pp. 105-110.

93. Chen Ch, Zhou W, Liu S, Deng Y, Cai F, et al. (2012) Increased NFKB signaling upregulates BACE1 expression and its therapeutic potential in Alzheimer's disease. Int j Neuropsychopharmacol 15(1): 77-90.

94. Liu CC, Kanekiyo T, Xu H, Bu G (2013) Apolipoprotein E and Alzheimer disease risk, mechamisms, and therapy. Nat Rev Neurol 9(2): 106-118.

95. Ahn KS, Aggarwal BB (2005) Transcription factor NFKB; a sensor for smoke and stress signals. Ann NY Acad Sci 1056(1): 218-33.

96. Conejero Goldberg C, Hyde TM, Chen S, Herman MM, Kleinman JE, et al. (2015) Cortical transcriptional profiles in APOE4 carriers with Alzheimer's disease: patterns of protection and degeneration. J Alzheimer Dis 48(4): 969-978.

97. Kim MK, Chung SW, Kim DH, Kim JM, Lee EK, et al. (2010) Modulation of NFKB activation by dietary zingerone vai MAPK pathway. Exp Gerontol 45(6): 419-426.

98. Jeong JJ, woo JY, Kim KA, Han MJ, Kim DH (2015) Latobacillus pentosus var plantarum C29 ameliorates age dependent memory impairment in Fischer rats. Lett Appl Microbiol 60(4): 307-314.

99. Rehman SU, Shah SA, Al T, Chung JI, Kim MO (2017) Anthocyanins reversed D-galactose induced oxidative stress and neuroinflammation 
mediated cognitive impairment in adult rats. Mol Neurobiol 54(1): 255271.

100. Kawahara TL, Michishita E, Adler AS, Damian M, Berber E, et al. (2009) SIRT6 links histone H\# lysine deacetylation to NFKB dependent gene expression and organismal life span. Cell 136(1): 62-74.

101. Sadagurski M, Cady G, Miller RA (2017) Anti-aging drugs reduce hypothalamic inflammation in a sex specific manner. Aging Cell 16(4): 652-660.

102. Pan H, Wang H, Zhu Lm, Mai L (2012) The absence of Nrf2 enhances NFKB dependent inflammation following scratch injury in mouse primary cultured astrocytes. Mediators Inflamm.

103. Frakes AE, Ferraiuolo I, Haidet Phillips AM, Schmelzer L, Braun L, et al. (2011) Microglia induce motor neuron death via the classical NFKB pathway in amyotrophic lateral sclerosis. Free Radic Biol znruton 81(5): 1009-1023.

104. Neymotin A, Calingsan NY, Wille E, Naseri N, Petri S et al. (2011) Neuroprotective effect of NRF2/ARE activators, CDDO ethylamide and CDDO trifluoroethylamide in mouse model of amyotrophic lateral sclerosis. Free Radic Biol Med 51(1): 88-96.

105. Kim JE. You DJ, Lee C, Ahn C, Seong JY, et al. (2010) Suppression of NFKB signaling by KEAP1 regulation of IKKbeta activity through autophagic degradation and inhibition of phosphorylaition. Cell Signal 22(11): 1645-1654.

106. Rada P, Rojo AI, Chowdhry S, McMahon M, Hayes JD et al. (2011) SCF/beta-TrCP promotes glycogen synthase kinase 3 dependent degradation of NRF2 transcription factor in a Keap1 independent manner. Mol Cell Biol 31(6): 1121-1133.

107. Chowdry S, Zhang Y, McMahon M, Sutherland C, Cuadrado A, et al. (2013) Nrf2 is controlled by two distinct beta TrCP recognition motifs in its Neh6 domain, one of which can be modulated by GSK-3 activity. Oncogne 32: 3765-3781.

108. Park SH, Park Min KH, Chen J, Hu X, Ivashkiv LB (2011) Tumor necrosis factor induces GSK3 kinase mediated cross tolerance to endotoxin in macrophages. Nat Immunol 12(7): 607-615.
109. Lee J, Kin MS (2007) The role of GSK3 in glucose homeostasis and the development of insulin resistance. Diabetes Res Clin Pract 779(1): S49-557.

110. Winston JT, Stack P, Beer Romero P, Chu CY, Elledge SJ, et al. (1999) The SCFbeta-TrCP-ubiquitin ligase complex associates specifically with phosphorylated destruction motifs in IKappaB $\alpha$ and beta catenin and stimulates IKappaB $\alpha$ ubiquitination in vitro. Genes Dev 13(3): 270283.

111. Ichimura Y, Waguri S, Sou Y, Kageyama S, Hasegawa J, et al. (2013) Phosphorylation of p62 activates the Keap-1-Nrf-2 pathway during selective autophagy. Mol Cell 51(5): 618-631.

112. Wooten MW, Geetha T, Seibenhener MI, Babu JR, Diaz Meco, et al. (2005) The p62 scaffold regulates nerve growth factor-induced NFKB activation by influencing TRAF6 polyubiquitination. J Biol Chemistry 280(42): 35625-35629.

113. Wardyn JD, Ponsford AH, Sanderson CM (2015) Dissecting molecular cross-talk between Nrf2 and NFKB response pathways. Biochem Soc Trans 43(4): 621626

114. Rojo, AI, Pajares P, Rada A, Nuñez A, Nevado Holgado AJ et al. (2017) NRF2 deficiency replicates transcriptomic changes in Alzheimer's patients and worsens APP and TAU pathology. Redox Biol 13: 444-451.

115. Schulze Topphoff U, Varrin Doyer M, Pekarek K, et al. (2016) Dimethyl fumarate treatment induces adaptive and innate immune modulation independent of NRF-2. Proc Natl Acad Sci USA 113(17): 4777-4782.

116. Wilms H, Sievers J, Rickert U, Rostami Yazdi M, Mrowietz U, et al. (2010) Dimethylfumarate inhibits microglial and astrocytic inflammation by suppressing the synthesis of nitric oxide, IL-1beta, TNF- $\alpha$ and IL- 6 in an in-vitro model of brain inflammation. J Neuroinflammation 7: 30 .

117. Albrecht P, Bouchachia N, Goebels N, Henke N, Hofstetter HH, et al. (2012) effects of dimethyl fumarate on neuroprotection and immunomodulation. J. Neuroinflammation 9: 163.

118. Fugita Y, Yamashita T (2018) Sirtuins in Neuroendocrine Regulation of Neurological Diseases. Front Neurosci 12: 778. 